\title{
THE UNEXPLORED LANDSCAPE OF TWO-BODY RESONANCES*
}

\author{
NATHANiEL CRAig
}

Department of Physics, UC Santa Barbara, Santa Barbara, CA 93106, USA

PATRICK DRAPER

Department of Physics, University of Massachusetts, Amherst, MA 01003, USA

KYOUNGCHUL KONG

Department of Physics and Astronomy, University of Kansas

Lawrence, KS 66045, USA

and

Pittsburgh Particle Physics, Astrophysics, and Cosmology Center

Department of Physics and Astronomy, University of Pittsburgh

Pittsburgh, PA 15260, USA

\section{YVonne NG, DANiel Whiteson}

Department of Physics and Astronomy, UC Irvine, Irvine, CA 92627, USA

(Received November 8, 2018; accepted April 3, 2019)

We propose a strategy for searching for theoretically-unanticipated new physics which avoids a large trials factor by focusing on experimental strengths. Searches for resonances decaying into pairs of visible particles are experimentally very powerful due to the localized mass peaks and have a rich history of discovery. Yet, due to a focus on subsets of theoreticallymotivated models, the landscape of such resonances is far from thoroughly explored. We survey the existing set of searches, identify untapped experimental opportunities and discuss the theoretical constraints on models which would generate such resonances.

DOI:10.5506/APhysPolB.50.837

\footnotetext{
* Funded by $\mathrm{SCOAP}^{3}$ under Creative Commons License, CC-BY 4.0.
} 


\section{Introduction}

Searches for two-body decays of heavy resonances have a rich history of important discoveries, from the $J / \psi$ to the Higgs boson. Such resonances can provide an unambiguous signature of a localized invariant mass peak and offer simple background estimation from sidebands, allowing for discovery without requiring full models of the signal or background processes. These experimental features, combined with compelling theoretical arguments, motivate much of the current program of resonance searches.

The theoretical arguments for new resonances mostly consist of simple generic extensions to the Standard Model (e.g. a new $\mathrm{U}(1)$ ) or modifications to the SM which address an outstanding theoretical problem (e.g. KaluzaKlein gravitons). To date, most of the experimental searches have followed these theoretical arguments, leading to many searches for pairs of identical objects (e.g. ee, $\mu \mu, j j$ ) and, in rarer cases, for non-identical pairs (e.g. $e \mu, Z W)$. However, the dramatic scale of the open theoretical questions facing particle physics suggests that a correct theory of Nature may not be one of the models currently in fashion or under specific consideration. This motivates an experimental program which is not narrowly focused on current models and the signatures they suggest, but with a broad scope and systematic approach capable of theoretically unanticipated discoveries. While there have been many proposals for model-independent search programs at hadron colliders (such as the framework of on-shell effective theories [1]), they have been largely motivated by specific theoretical frameworks and, consequently, many holes remain in the existing experimental program at the LHC. To make concrete progress, we propose a systematic search for new particles decaying into $n$-body resonances. In the $n=2$ case, this would consist of searches for resonances in all pairs of objects, even those which have no theoretical motivation or are theoretically disfavored.

The typical difficulty facing searches without specific theoretical motivation is the large number of possible observables, which incurs a very large trials factor and greatly reduces the discovery sensitivity. Here, rather than relying on theoretical guidance, we propose to restrict the vast space of possible theories into those that align well with experimental strengths. We are interested in covering the intermediate ground between the very specific and the very general search programs, by focusing on well-defined topologies independent of specific theory considerations. This broadens the search program beyond favored theories, but not so much so as to compromise discovery potential. Given that the data exist and resonances are fairly easy to discover, we argue that the two-particle spectra are worth directly examining. In many cases, there are indirect constraints on such resonances from other experiments or subjective theoretical arguments, but there is no real substitute for a direct search. 
In this paper, we lay out the details of the implementation of such a search program and survey the existing experimental and theoretical landscape for exclusive $n=2$-body resonances, leaving $n=3+$ (as well as inclusive $n=2$ final states) for future work. We find that the majority of 2-body resonances have some indirect theoretical constraints but have received almost no experimental attention, leaving most of the landscape unexplored and a large potential for unanticipated discovery.

\section{Scope and experimental searches}

We consider resonances decaying to a basic set of identifiable light objects (charged leptons, photons, light-quark jets, $b$-tagged jets) as well as heavy objects (top quarks, weak bosons, Higgs bosons) which are routinely identified $^{1}$. In the case of $n=2$ objects, this gives 55 unique pairs of exclusive final states, see Table I. Final states with higher number of objects have a larger number of exclusive final states; we reserve these for future work.

We examined experimental searches from ATLAS and CMS in data collected from proton-proton collisions with $\sqrt{s}=8 \mathrm{TeV}$. We consider exclusive final states only in terms of the pairs of identifiable objects defined above. For example, in the $e \gamma$ category of this exclusive $n=2$ survey, we consider only searches for $e \gamma$, of which there are none, and do not consider searches for $e^{+} e^{-} \gamma$, of which there are several motivated by excited lepton models that give a resonance in $e \gamma$. The final state of $e^{+} e^{-} \gamma$ would be covered by an $n=3$ study, and extrapolation of those limits to the $n=2 \mathrm{er}$ category requires theoretical assumptions about the production modes.

The survey of $n=2$ final states is shown in Table I, with the striking feature that most diagonal entries have existing searches, whereas most offdiagonal entries do not. In the case of the Higgs boson in particular, there are several unexamined resonance categories. Note that the lack of searches in these resonance categories is not for want of theory models. Examples of theories that populate the entire landscape of 2-body resonances are shown in Table II.

Even in cases where searches exist, there are often unexamined regions in the resonance mass. Figures 1 and 2 show the strongest limits on the cross section times branching ratio as a function of the resonance mass for all results which satisfy the requirements.

\footnotetext{
${ }^{1}$ One could imagine restricting the scope to light objects, categorizing the heavy objects as higher-level decays (e.g. $X \rightarrow W W \rightarrow 4 j$ would be considered in the $n=4$ category rather than $X \rightarrow W W$ as $n=2$ ). This is equivalent, but allows us to call attention to these typical objects rather than considering them as special mass cases of higher-level decays.
} 


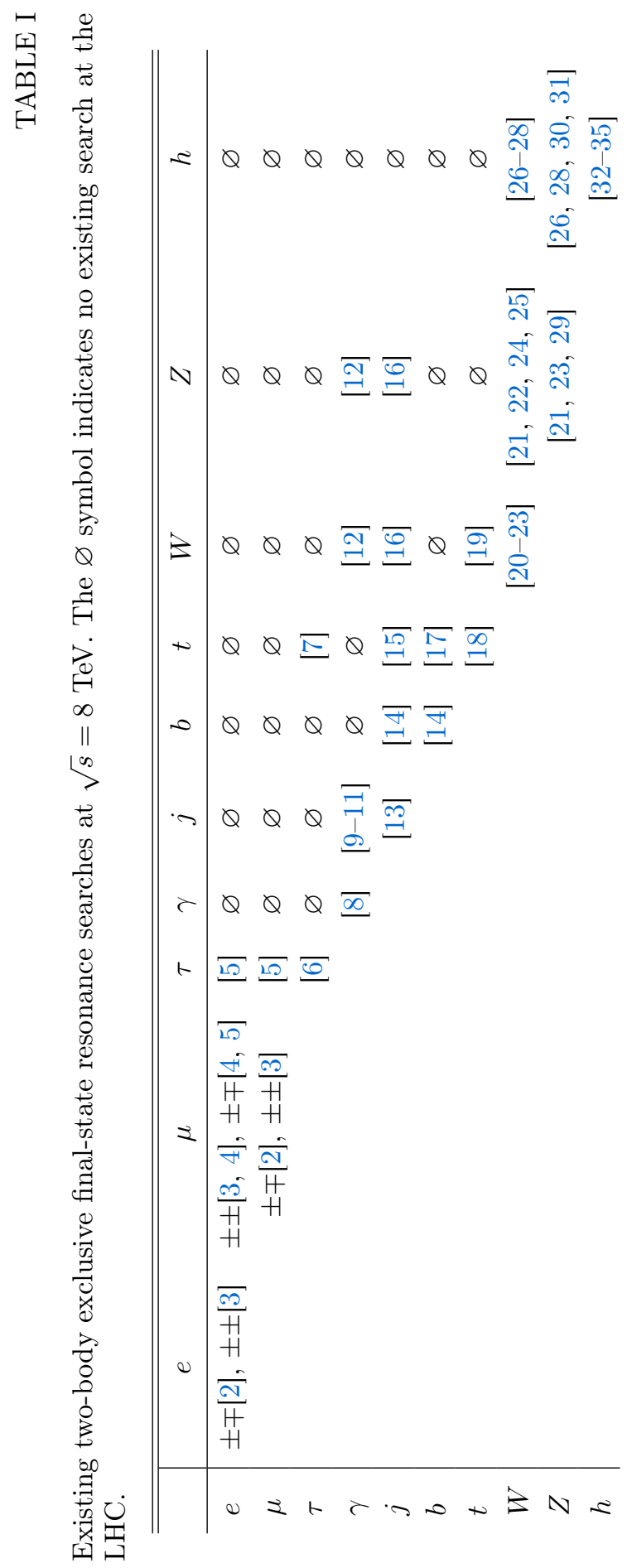


The Unexplored Landscape of Two-body Resonances

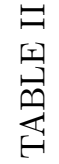

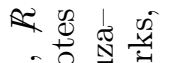

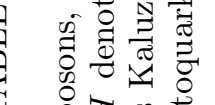

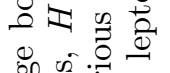

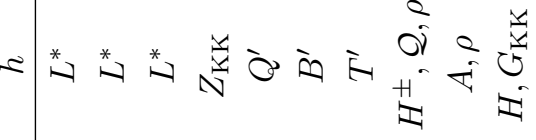

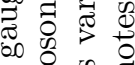

శే]

. 잉

记四四

च च के क्ष

范的.

엉 च

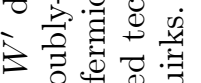

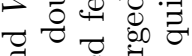

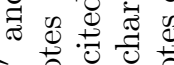

స $\left.\begin{array}{lll}0 \\ 0\end{array}\right)$

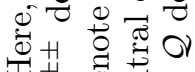

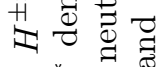

过

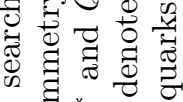

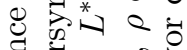

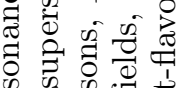

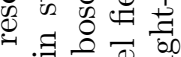

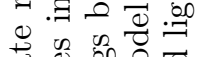

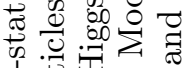

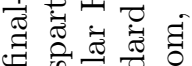

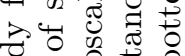

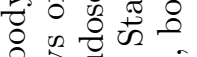

1

过 20

$\infty 00000$

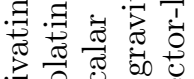

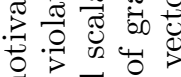

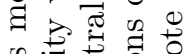

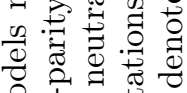

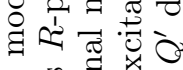

$\Rightarrow$ है 00 व

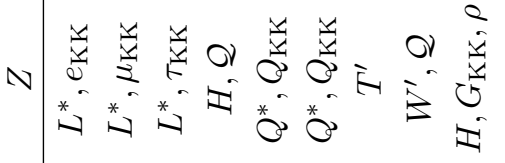

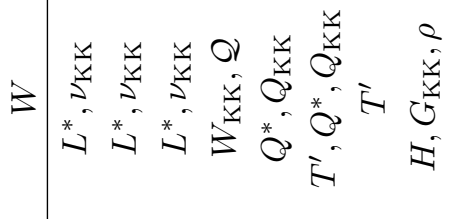

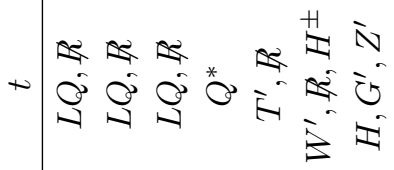

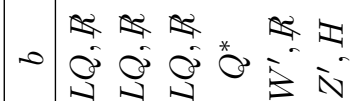

\& 0 茟

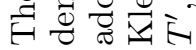

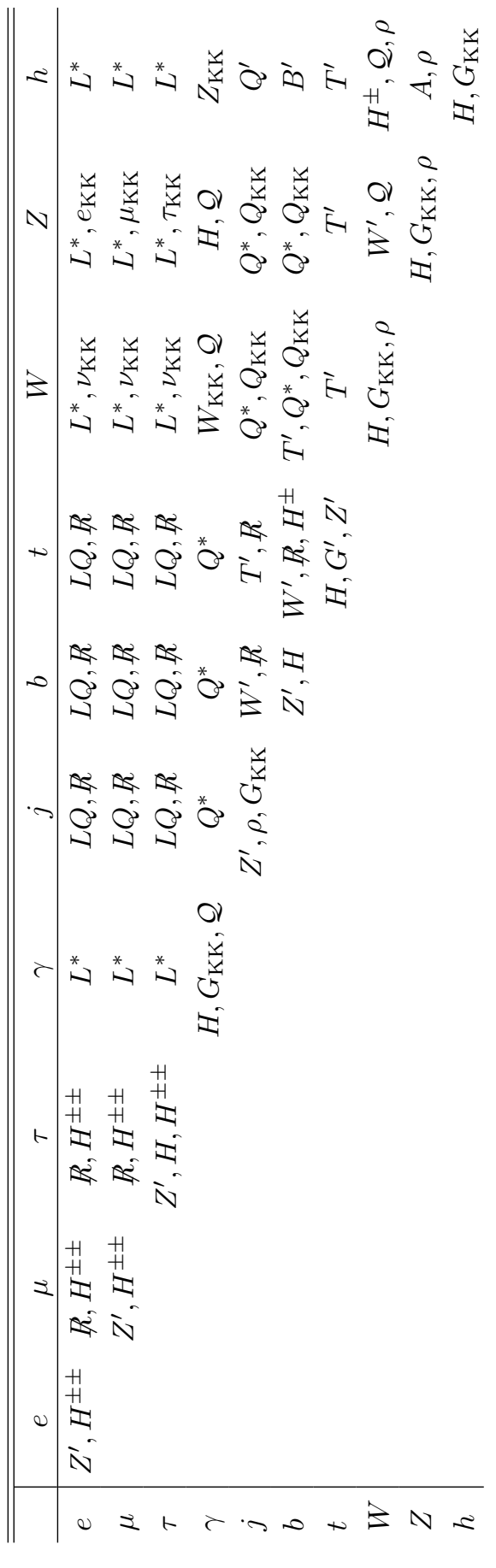



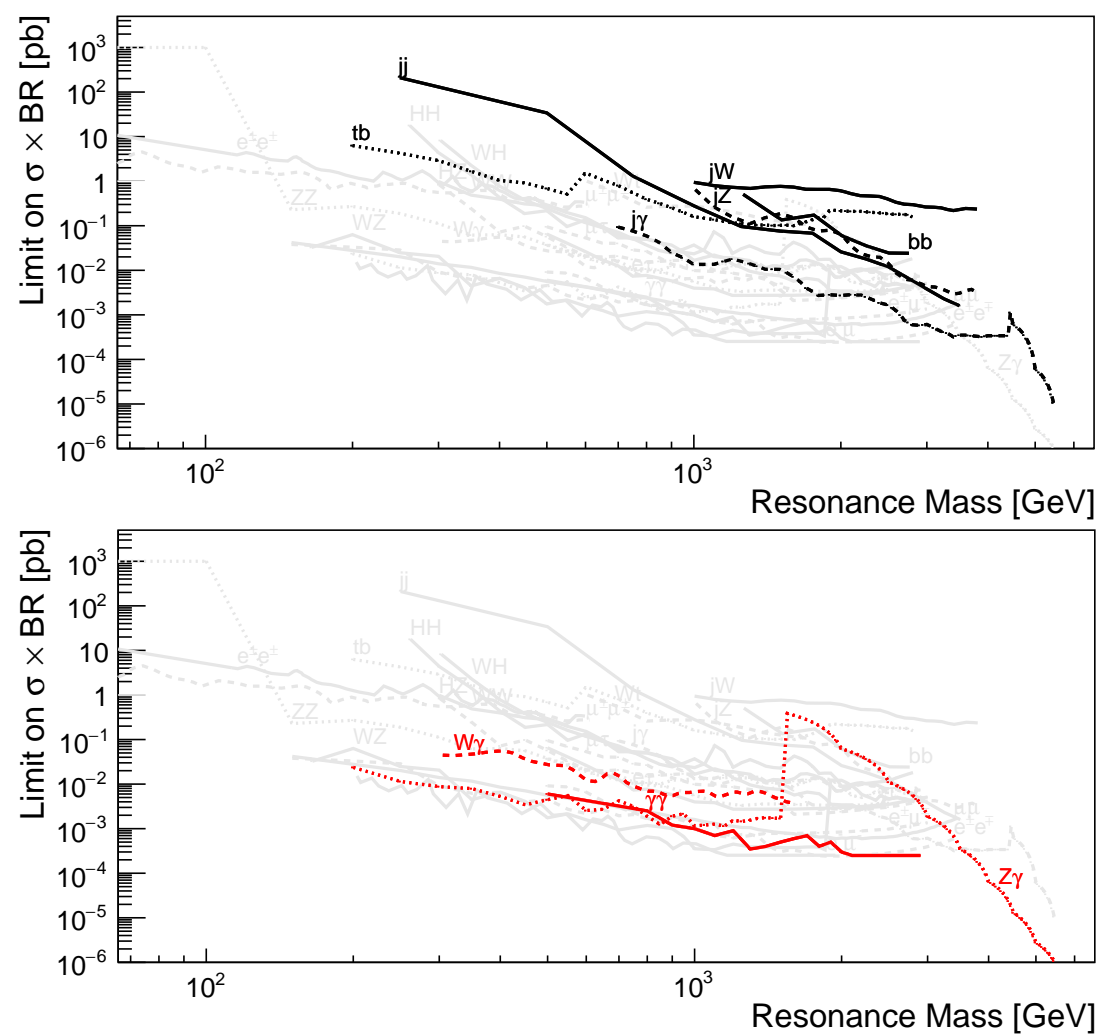

Fig. 1. Existing limits on the cross section times branching ratio for resonances to various 2-body final states, as a function of the resonance mass. Top panel emphasizes hadronic final states, bottom panel emphasizes photonic final states. References for searches can be found in Table I.

\section{Theoretical constraints}

Various theoretical constraints may be imposed on $n$-body resonances, which, in turn, influence the likely production and decay modes at the LHC. In order to maintain the broadest possible scope, we consider only the most stringent constraints imposed by gauge invariance and Lorentz invariance, as experimental constraints on e.g. flavor violation depend on the details of the underlying model and may in principle be evaded.

Gauge invariance and Lorentz invariance restrict the possible statistics and quantum numbers of a resonance decaying to a specified 2-body final state. The statistics and possible $\mathrm{SU}(3)_{\mathrm{c}}$ and $\mathrm{U}(1)_{\mathrm{em}}$ numbers of 2-body resonances are enumerated according to their exclusive final state in Table III. 

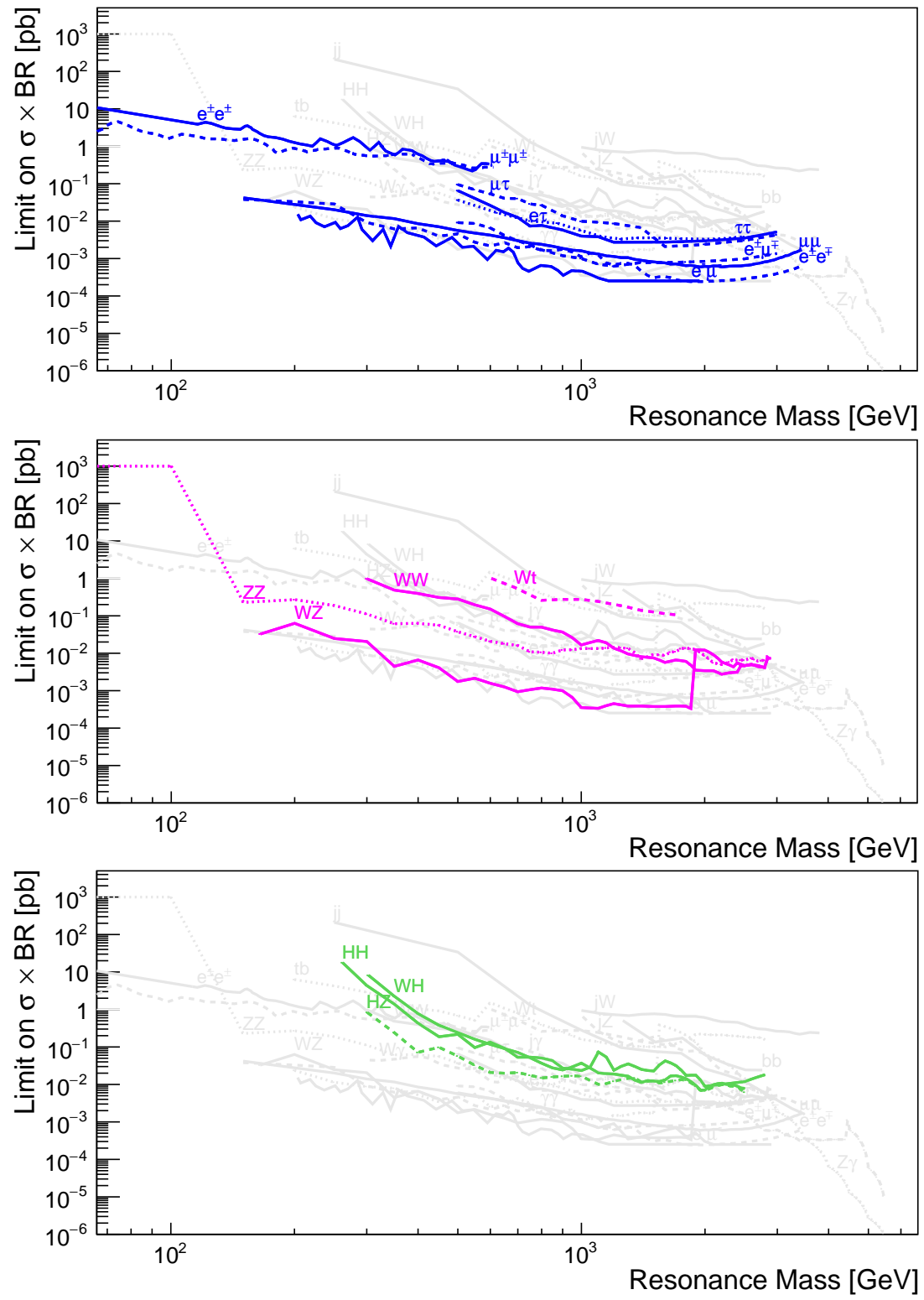

Fig. 2. Existing limits on the cross section times branching ratio for resonances to various 2-body final states, as a function of the resonance mass. The top panel emphasizes leptonic final states, the center panel emphasizes bosonic final states, and the bottom panel emphasizes Higgs final states. References for searches can be found in Table I. 


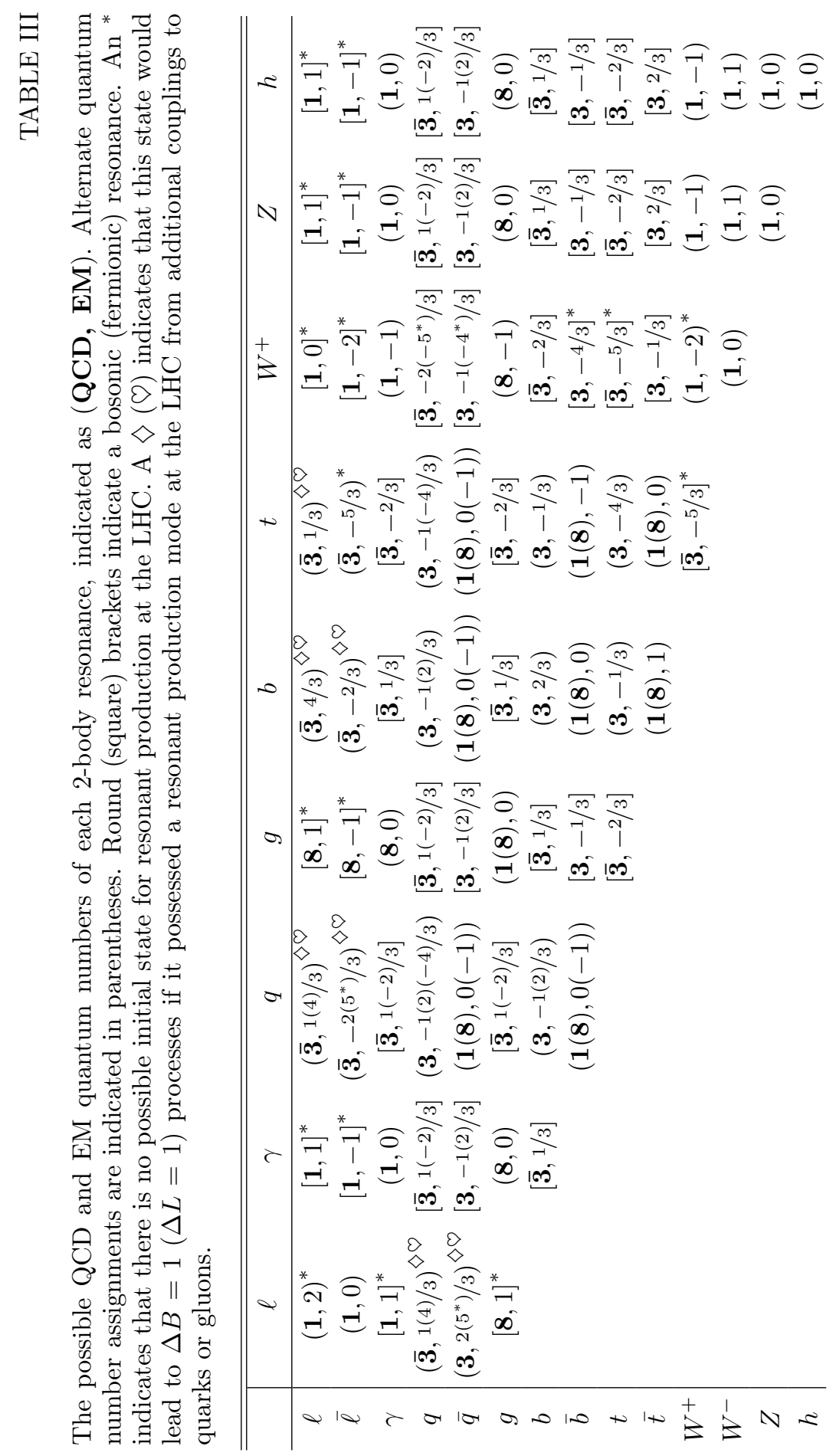


Note that we enumerate only $\mathrm{SU}(3)_{\mathrm{c}} \times \mathrm{U}(1)_{\mathrm{em}}$ quantum numbers rather than $\mathrm{SU}(3)_{\mathrm{C}} \times \mathrm{SU}(2)_{\mathrm{L}} \times \mathrm{U}(1)_{\mathrm{Y}}$ quantum numbers, because a large number of $\mathrm{SU}(3)_{\mathrm{C}} \times \mathrm{SU}(2)_{\mathrm{L}} \times \mathrm{U}(1)_{\mathrm{Y}}$ representations may share the same exclusive final state provided additional insertions of the Higgs vacuum expectation value. We also do not exhaustively list all possible $\mathrm{SU}(3)_{\mathrm{c}}$ representations, but for simplicity, restrict our attention to states transforming in the fundamental or adjoint representation; resonances transforming in other representations of $\mathrm{SU}(3)_{\mathrm{c}}$ may have different pair production cross sections but do not lead to significantly different signatures. While a fermionic resonance with the Standard Model quantum numbers generally contributes to gauge anomalies, these anomalies may be canceled by additional particles that do not influence the collider signatures of the resonance.

Gauge invariance and Lorentz invariance also dictate the structure of operators coupling a resonance to the Standard Model particles, and in many cases, the couplings must arise via irrelevant operators. For example, a resonance $X$ decaying to $t g$ cannot couple via a minimal gauge coupling $\bar{X} \gamma^{\mu} G_{\mu} t$, but may couple via e.g. a chromoelectric dipole operator of the form of $\bar{X} \gamma^{\mu \nu} G_{\mu \nu} t$. In many cases, more than one Lorentz structure is allowed for a given coupling. The various possible Lorentz structures for each coupling have a modest impact on kinematic distributions for the production and decay of each resonance (see e.g. [1]), but they do not alter the key feature of interest in this work, namely a bump in the $n$-body invariant mass spectrum.

Note that these conclusions may be altered in the presence of significant interference effects, which may lead to deficits or peak-dip structures in the invariant mass spectrum if the Standard Model continuum interferes with the signal process. The existence and structure of interference effects cannot be determined by quantum numbers alone, and depends additionally on both the Lorentz structure and phases of couplings between the resonance and the Standard Model states. However, in the limit of weak coupling, interference between a narrow resonance and the Standard Model continuum backgrounds is negligible and may be neglected. To good approximation, as an expansion at weak coupling, searches for $n$-body resonances may, therefore, be parameterized solely in terms of the resonance mass, width, and production cross section times branching ratio.

Having specified the possible gauge quantum numbers of the 2-body resonance given the final state, gauge invariance and Lorentz invariance provide a guide to the possible production modes at the LHC. For each resonance there are three possibilities:

1. The particle can be resonantly produced either exclusively using its tree-level decay coupling (as in, e.g., a resonance decaying to $q q$ or $g g$ ); via loop-induced processes involving the decay coupling (as in, 
e.g., gluon fusion production of a $t \bar{t}$ resonance); or via additional couplings to quarks and gluons allowed by its quantum numbers. The presence of such additional couplings may lead to additional theoretical constraints discussed below. Such resonant production channels fall under the scope of the exclusive 2-body searches proposed here.

2 . The particle can be produced via associated production exclusively using its decay couplings. For example, a resonance $X$ coupling to $t W^{+}$can be produced in the process $q g \rightarrow t q X$ using only the $X t W^{+}$ coupling and Standard Model gauge couplings. This assumes no additional couplings to quarks and/or gluons. Such associated production channels fall under the scope of $n \geq 3$ studies, with a feature in the appropriate 2-body invariant mass spectrum.

3. The particle can be pair produced using its gauge quantum numbers (e.g. Drell-Yan via electroweak quantum numbers). This process is kinematically suppressed for heavier resonances, but may be appreciable if the gauge couplings are significantly larger than the decay couplings. Such pair production channels fall under the scope of $n=4$ studies, with features in the appropriately-paired 2-body invariant mass spectra.

The possible production modes for each resonance are enumerated in Table IV. In principle, a given resonance may be produced in all three modes, with varying rates depending on the relative sizes of phase space factors and production and decay couplings. In each case, the final state contains a peak in the appropriate 2-body invariant mass, but with varying amounts of additional event activity. In this sense, the associated- and pair-production modes may not qualify for the $n=2$ exclusive case considered above, but serve as a useful foundation for future $n>2$ studies.

As is apparent in Table IV, there are several possible 2-body resonances for which resonant production is incompatible with the Standard Model gauge invariance, in the sense that the quantum numbers of the final state cannot be produced by any initial state with appreciable parton density in proton-proton collisions. Nonetheless, searches for these 2-body resonances at the LHC remain motivated by the possibility of new physics that mimics a Standard Model final state in the LHC detectors (in the sense that, e.g., a long-lived neutral particle decaying to electron-positron pairs might be reconstructed as a photon). These states may also be produced in associated production with associated particles sufficiently soft to still appear as an exclusive 2-body resonance, or may originate from $n \geq 2$ exclusive final states with missing energy appearing in $n=2$ exclusive searches. Such states may also be resonantly produced at other colliders consistent with gauge invariance, such as in electron-proton collisions at HERA. 

\& \& \& \& \& \& \& \& \& \& \& \&

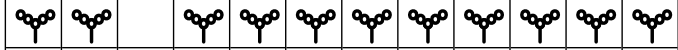

N

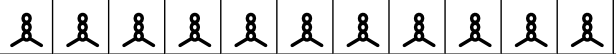

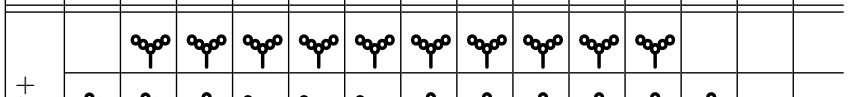

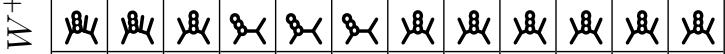

\&之\&\&之 \& \&

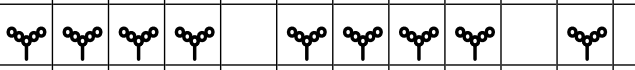

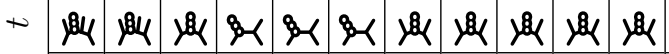

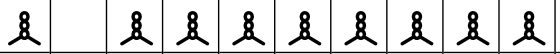

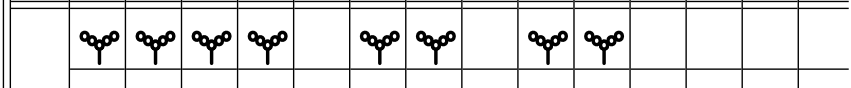

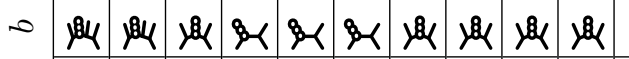

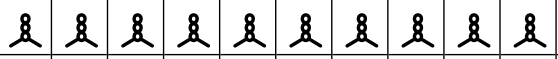

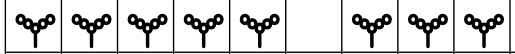

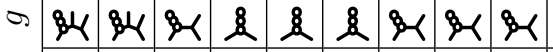

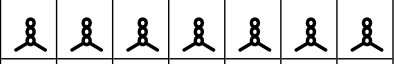

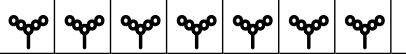

O 以 K K \& \& \& $K$

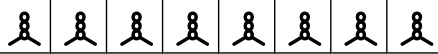

포으.

$\tau$.

ฮु

卷

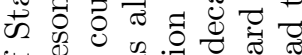

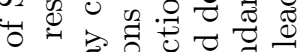

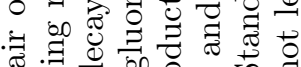

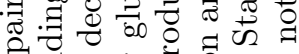

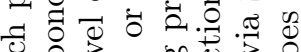

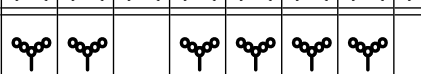

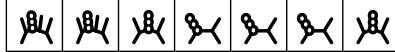

손소논

$\infty$ क $\infty$ क

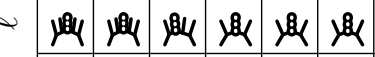

$$
\text { \& \& \& }
$$


Apart from gauge invariance and Lorentz invariance, less robust constraints may also apply. Many such constraints arise only when the resonance possesses both its decay coupling and additional couplings to quarks and/or gluons. Proton decay provides the strongest such constraint, as strong bounds on the proton lifetime imply that the couplings of resonances inducing proton decay are vanishingly small. In the case of 2-body resonances, resonances coupling to a single pair of the Standard Model particles will not induce proton decay, but proton decay may be induced by additional couplings to quarks required for resonant production at the LHC. Resonances for which this occurs are indicated in Table III; in these cases, it is reasonable to expect $n=2$ resonant production rates to be small.

Beyond proton decay, there are a variety of constraints on flavor violation, lepton number violation, and other types of baryon number violation but, in practice, even strong constraints may be avoided by appropriate symmetries, textures, or fortuitous cancellations (as in e.g. maximal flavor violation [37] or diquark-type interactions [38]). In these cases, there is no substitute for a direct search.

\section{Exploring the landscape}

We have presented a survey of the landscape of two-body resonance searches, revealing that a large number of cases have received no experimental attention. Given the power of the LHC experiments to discover resonances, we find this to be a compelling argument for searches. While theoretical guidance is of great value, it should not completely determine the course of experimental efforts.

Indeed, we expect these searches to be fairly straightforward. Without loss of generality, we use the $e \gamma$ final state as an example. The first step would be the identification of a suitable trigger, in this case likely a singleobject electron or photon trigger, followed by the selection of collisions which contain exactly one electron and exactly one photon which meet standard experimental reconstruction criteria.

The greatest challenge is in constructing a model for the Standard Model background, which is precisely what motivates our focus on searching for narrow resonances. There are a suite of well-worn techniques [39-41] to fit parametric models to smooth backgrounds, as well as new non-parametric approaches [42] that can describe a broader set of smooth background distributions under a localized peak. What remains is to provide a hypothesis for the signal shape. This may be a generic Gaussian bump, or a more physical curve generated by a candidate hypothesis, or even more model-independent approaches [42]. Critically, however, a theoretically-motivated hypothesis with a reasonably large production cross section is not a requirement; the observation of a significant peak in the $e \gamma$ spectrum would certainly lead to the rapid generation of candidate models to explain it. 


\section{Discussion}

The data from the LHC are extraordinarily valuable, in that its collection required an enormous investment of financial and human resources and in its potential power to answer outstanding questions of particle physics. However, once those resources are spent and the data are collected, there remain difficult questions regarding how to use it. Experimental analysis of a given final state requires limited human and financial resources, and every search increases field-wide trials factor, making any local excess less globally significant. Therefore, it is necessarily the case that some experimental territory will be left uncovered, and proposals for new experimental searches must have a compelling argument.

Here, we have argued that in addition to the usual stable of theoreticallymotivated searches, a set of experimentally-motivated searches should be conducted. We propose a set of exclusive 2-body resonance searches, which naturally limits the number of final states and is well-matched to experimental capabilities. This is in contrast to the strategy of general searches, which attempt to satisfy a broad set of theory motivations, but do not focus on experimental strengths and suffer a very large trials factor.

The final states with matched objects have been examined, though there remain openings at low- and high-mass regions. More significantly, we find that many of the mismatched pair final states have had no attention, despite the existence of theoretical models and the absence of strong theoretical constraints.

We thank Mohammad Abdullah, Jahred Adelman, and Tim Tait for useful conversations. This research was supported in part by the National Science Foundation under grant No. NSF PHY11-25915 and the U.S. Department of Energy under grants DE-SC0014129 and DE-FG02-12ER41809; the authors are grateful to the Kavli Institute for Theoretical Physics, where some of this work was done.

\section{REFERENCES}

[1] N. Arkani-Hamed et al., arXiv:hep-ph/0703088.

[2] G. Aad et al. [ATLAS Collaboration], Phys. Rev. D 90, 052005 (2014) [arXiv:1405.4123 [hep-ex]].

[3] G. Aad et al. [ATLAS Collaboration], J. High Energy Phys. 1503, 041 (2015) [arXiv: 1412.0237 [hep-ex]].

[4] V. Khachatryan et al. [CMS Collaboration], Eur. Phys. J. C 76, 317 (2016) [arXiv:1604.05239 [hep-ex]]. 
[5] G. Aad et al. [ATLAS Collaboration], Phys. Rev. Lett. 115, 031801 (2015) [arXiv:1503.04430 [hep-ex]].

[6] G. Aad et al. [ATLAS Collaboration], J. High Energy Phys. 1507, 157 (2015) [arXiv:1502.07177 [hep-ex]].

[7] V. Khachatryan et al. [CMS Collaboration], J. High Energy Phys. 1507, 042 (2015) [arXiv:1503.09049 [hep-ex]].

[8] G. Aad et al. [ATLAS Collaboration], Phys. Rev. D 92, 032004 (2015) [arXiv: 1504.05511 [hep-ex]].

[9] V. Khachatryan et al. [CMS Collaboration], Phys. Lett. B 738, 274 (2014) [arXiv:1406.5171 [hep-ex]].

[10] G. Aad et al. [ATLAS Collaboration], Phys. Lett. B 728, 562 (2014) [arXiv:1309.3230 [hep-ex]].

[11] G. Aad et al. [ATLAS Collaboration], arXiv:1512.05910 [hep-ex].

[12] G. Aad et al. [ATLAS Collaboration], Phys. Lett. B 738, 428 (2014) [arXiv: 1407.8150 [hep-ex]].

[13] G. Aad et al. [ATLAS Collaboration], Phys. Rev. D 91, 052007 (2015) [arXiv:1407.1376 [hep-ex]].

[14] Technical Report CMS-PAS-EXO-12-023, CERN, Geneva 2013.

[15] G. Aad et al. [ATLAS Collaboration], Phys. Rev. D 86, 091103 (2012) [arXiv:1209.6593 [hep-ex]].

[16] V. Khachatryan et al. [CMS Collaboration], J. High Energy Phys. 1408, 173 (2014) [arXiv:1405.1994 [hep-ex]].

[17] G. Aad et al. [ATLAS Collaboration], arXiv:1512.03704 [hep-ex].

[18] G. Aad et al. [ATLAS Collaboration], J. High Energy Phys. 1508, 148 (2015) [arXiv:1505.07018 [hep-ex]].

[19] G. Aad et al. [ATLAS Collaboration], arXiv:1510.02664 [hep-ex].

[20] G. Aad et al. [ATLAS Collaboration], J. High Energy Phys. 1601, 032 (2016) [arXiv:1509.00289 [hep-ex]].

[21] G. Aad et al. [ATLAS Collaboration], J. High Energy Phys. 1512, 055 (2015) [arXiv:1506.00962 [hep-ex]].

[22] G. Aad et al. [ATLAS Collaboration], Eur. Phys. J. C 75, 209 (2015) [Erratum ibid. 75, 370 (2015)] [arXiv:1503.04677 [hep-ex]].

[23] V. Khachatryan et al. [CMS Collaboration], J. High Energy Phys. 1408, 174 (2014) [arXiv:1405.3447 [hep-ex]].

[24] V. Khachatryan et al. [CMS Collaboration], Phys. Lett. B 740, 83 (2015) [arXiv:1407.3476 [hep-ex]].

[25] G. Aad et al. [ATLAS Collaboration], Phys. Lett. B 755, 285 (2016) [arXiv: 1512.05099 [hep-ex]].

[26] G. Aad et al. [ATLAS Collaboration], Eur. Phys. J. C 75, 263 (2015) [arXiv:1503.08089 [hep-ex]].

[27] V. Khachatryan et al. [CMS Collaboration], arXiv:1601.06431 [hep-ex]. 
[28] V. Khachatryan et al. [CMS Collaboration], J. High Energy Phys. 1602, 145 (2016) [arXiv:1506.01443 [hep-ex]].

[29] G. Aad et al. [ATLAS Collaboration], Eur. Phys. J. C 76, 45 (2016) [arXiv:1507.05930 [hep-ex]].

[30] V. Khachatryan et al. [CMS Collaboration], Phys. Lett. B 748, 221 (2015) [arXiv:1504.04710 [hep-ex]].

[31] V. Khachatryan et al. [CMS Collaboration], Phys. Lett. B 748, 255 (2015) [arXiv: 1502.04994 [hep-ex]].

[32] G. Aad et al. [ATLAS Collaboration], Phys. Rev. D 92, 092004 (2015) [arXiv:1509.04670 [hep-ex]].

[33] V. Khachatryan et al. [CMS Collaboration], Phys. Lett. B 749, 560 (2015) [arXiv: 1503.04114 [hep-ex]].

[34] Technical Report CMS-PAS-EXO-15-008, CERN, Geneva 2015.

[35] V. Khachatryan et al. [CMS Collaboration], Eur. Phys. J. C 76, 371 (2016) [arXiv:1602.08762 [hep-ex]].

[36] K. Kong, Non-standard resonance searches at the LHC, http://goo.gl/VYEb4R, Accessed: 2016-08-01.

[37] S. Bar-Shalom, A. Rajaraman, Phys. Rev. D 77, 095011 (2008) [arXiv:0711.3193 [hep-ph]].

[38] G.F. Giudice, B. Gripaios, R. Sundrum, J. High Energy Phys. 1108, 055 (2011) [arXiv: 1105.3161 [hep-ph]].

[39] UA2 Collaboration, Z. Phys. C 49, 17 (1991).

[40] G. Aad et al. [ATLAS Collaboration], Phys. Rev. Lett. 108, 111803 (2012) [arXiv:1202.1414 [hep-ex]].

[41] S. Abrahamyan et al. [APEX Collaboration], Phys. Rev. Lett. 107, 191804 (2011) [arXiv:1108.2750 [hep-ex]].

[42] M. Frate et al., arXiv:1709.05681 [physics.data-an]. 